\title{
Identification of the Rice Blast Resistance Gene Pib in the National Small Grains Collection
}

\author{
M. RoyChowdhury, Y. Jia, M. H. Jia, R. Fjellstrom, and R. D. Cartwright
}

First and fifth authors: Cell and Molecular Biology Program, University of Arkansas, Fayetteville 72701; and second, third, and fourth authors: U.S. Department of Agriculture-Agricultural Research Service, Dale Bumpers National Rice Research Center (DB NRRC), Stuttgart, AR 72160.

Accepted for publication 6 April 2012.

\begin{abstract}
RoyChowdhury, M., Jia, Y., Jia, M. H., Fjellstrom, R., and Cartwright, R. D. 2012. Identification of the rice blast resistance gene Pib in the National Small Grains Collection. Phytopathology 102:700-706.

The $P i b$ gene in rice confers resistance to a wide range of races of the rice blast pathogen, Magnaporthe oryzae, including race IE1k that overcomes Pita, another broad-spectrum resistance gene. In this study, the presence of $P i b$ was determined in 164 rice germplasm accessions from a core subset of the National Small Grains Collection utilizing DNA markers and pathogenicity assays. The presence of $P i b$ was evaluated

(Pib-dom) derived from the Pib gene sequence. Pathogenicity assays using two avirulent races (IE1k and IB1) and a virulent race (IB54) were performed to verify the resistance responses of accessions. Of the 164 accessions evaluated, 109 contained the Pib gene as determined using both SSR markers and pathogenicity assays, albeit different haplotypes were detected. The remaining 52 germplasm accessions were different in their responses to the blast races IB54, IE1k, and IB1, thus indicating the presence of $R$ gene(s) other than $P i b$. The accessions characterized in this study could be used for marker-assisted breeding to improve blast resistance in indica and japonica cultivars worldwide.
\end{abstract} with two simple sequence repeat (SSR) markers and a dominant marker
Rice blast caused by Magnaporthe oryzae B. Couch is the most destructive disease affecting rice production worldwide. The use of resistant cultivars has been the most economical and efficient method for controlling this disease. However, the lifespan of many resistant cultivars is only a few years, due to the loss of resistance in the face of hyper-variability of the pathogen $(20,28$, 31). The inheritance of major-gene-mediated resistance to the blast pathogen has been studied extensively worldwide. Major resistance $(R)$ genes are effective in preventing infection by races of $M$. oryzae containing the corresponding avirulence $(A V R)$ genes $(11,32)$. Presently, more than 70 blast $R$ genes have been identified, and 13 of them have also been characterized using molecular markers and subsequently used to develop resistant cultivars $(2,6$, 7,9,12-16,21-23,29,30,35,38,39).

Molecular markers tightly linked to major $R$ genes are useful for marker assisted selection (MAS). Although a large number of blast $R$ genes have been fine mapped based on closely linked markers and/or some of them cloned based on marker information, there are only a few published examples where the markers had a direct impact on plant breeding. DNA markers derived from the cloned $P i b$ and Pita blast $R$ genes are currently used in several rice breeding programs (18). Allele-specific DNA markers were also developed to distinguish Pikm and Pik (8). In addition, polymerase chain reaction (PCR)-based single nucleotide polymorphism markers for genes at the Piz locus are also known to be used in breeding programs (14) and linked markers have been successfully used to identify germplasm that carry $\mathrm{Piz}$ (30). The $R$ gene in rice lines, BL8, BL9, BL10, and BL11, was named by

Corresponding author: Y. Jia; E-mail address: yulin.jia@ars.usda.gov

http://dx.doi.org/10.1094/PHYTO-02-12-0026-R

This article is in the public domain and not copyrightable. It may be freely reprinted with customary crediting of the source. The American Phytopathological Society, 2012.
Kiyosawa (19) as $P i b$ and has been used extensively in rice breeding programs in Japan, China, and Indonesia (19,26,27,38). The gene encoding a cytoplasmic protein with a nucleotide binding site and leucine rich repeats (NBS-LRR) was the first cloned blast $R$ gene (35). The availability of a high-density linkage map (13) and DNA markers in the Pib region (27) has facilitated the identification of additional molecular markers more closely linked to Pib (10). Pib was introduced into the U.S. rice cultivar 'Saber' (25) from the indica 'Teqing', a cultivar from China and the gene has been identified in rice cultivars resistant to blast races IA45, IB1, IH1, IB45, IG1, IE1k, IC17, and IE1k (24,25). Resistance to race IE1k can be attributed to the presence of only Pib or Piz in U.S. rice cultivars. Race IB1 can be used to distinguish the presence of either of these genes because $\mathrm{Piz}$ provides resistance to IE1k, but is ineffective against IB1, whereas $P i b$ is effective against both.

The objectives of this study were to (i) identify the Pib gene in a core collection of 1,790 rice germplasm accessions, collected from 113 countries, representing an estimated $70 \%$ of the genetic diversity of the entire U.S. Department of Agriculture (USDA) National Small Grains Collection of rice germplasm (37), using previously identified simple sequence repeat (SSR) markers closely linked to the $P i b$ gene and a dominant marker derived from $P i b$; and (ii) determine disease reactions of the accessions containing Pib to differential U.S. blast races. The results indicated that the molecular markers served as an effective diagnostic tool for the presence of $P i b$.

\section{MATERIALS AND METHODS}

Plant materials. Prior to the purification of the collection by single seed descent (1), the USDA core collection consisting of 1,790 accessions was screened with SSR marker RM208 tightly linked to the $P i b$ gene and a dominant marker Pib-dom, derived from a portion of the Pib sequence (10). Four grams of seed from each accession of the purified core was provided by the Genetic 
Stocks Oryza Collection (GSOR, www.ars.usda.gov/spa/dbnrrc/ gsor) at Dale Bumpers National Rice Research Center (DB NRRC). Twelve seeds of each accession were germinated in 96 well inserts $(10 \times 20 \times 2 \mathrm{~cm})$ (Hummert International, MO). Prior to seeding, the inserts were placed in trays $(26.67 \times 53.34 \times 6.35$ $\mathrm{cm}$, Model INT0804, Hummert International) and filled with silt loam soil ( $\mathrm{pH} 5.5$ to 5.8) fertilized with Osmocote Pro 15-9-12 (Scotts-Sierra Horticultural Products Company, OH), autoclaved, and stored at $-20^{\circ} \mathrm{C}$ for 3 days. The trays were completely filled with water. Seedlings were grown in winter (November to December 2010) for 4 weeks in the greenhouse maintained at 23 to $29^{\circ} \mathrm{C}$ during the day and 22 to $25^{\circ} \mathrm{C}$ during the night until the three to four leaf stage, in preparation for pathogenicity assays and subsequent DNA extraction.

Pathogenicity assays. Pathogenicity assays were performed on 164 germplasm accessions confirmed by SSR markers as having $P i b$, and a positive control 'Saber' (PI 633624). The race IE1k detects the presence of $P i b$ and Piz; however, IB1 detects the presence of $\mathrm{Pib}$, not Piz (Fig. 1). Single spores of virulent (VIR) isolate (unnamed isolate-race IB54), AVR isolates TM2 (race IE1k), and an unnamed isolate-race IB1 of $M$. oryzae were selected for pathogenicity tests in the present study. There were four replicates for each germplasm accession. The presence of the $P i b$ gene in each accession was verified by the pattern of resistance or susceptibility to both AVR and VIR isolates. Pathogen inoculation was performed using a modified procedure based on Valent et al. (33). Briefly, plants were inoculated with $40 \mathrm{ml}$ of a spore suspension $\left(5 \times 10^{5}\right.$ spores $/ \mathrm{ml}, 0.25 \%$ gelatin $)$ using a hand atomizer connected to an air compressor $(100 \mathrm{kPa})$. Inoculated plants were maintained at approximately $95 \%$ relative humidity in a clear polyethylene autoclave bag $(24 \times 36 \mathrm{~cm}$ and $1.5 \mathrm{~mm}$ thick) at room temperature (Product code 018143, Fisher Scientific). Approximately $24 \mathrm{~h}$ after inoculation, removing the humidity chamber, plants were moved to the greenhouse for an additional 6 days. Disease reactions were assessed 7 days after inoculation using a visual rating scale from 0 to 5 , as previously described (30). For each accession, seven to eight seedlings were evaluated and each pathogenicity assay was conducted three times.

DNA extraction. DNA was extracted from bulked leaves from each of four replicates used in the pathogenicity assay using a rapid DNA extraction procedure (36). After extraction, sample DNA was prepared for PCR through a Biomek 2000 Lab Automation Work Station (Beckman and Coulter, Brea, CA) using manufacturer protocols.
DNA markers and analysis. Three SSR markers from which data are already available from the purified core collection (1) were used to screen current germplasm to preclude any seed mixtures or experimental error. The markers selected were RM224, RM171, and RM231 because all three markers are robust and have high PIC (polymorphism information content) value. Three markers previously identified as associated with the presence of Pib (10), RM208, Pib-dom, and RM166 were used to screen purified accessions from the core collection as verification of previous marker screen on unpurified accessions. Fluorescently labeled markers were analyzed by capillary electrophoresis based on the methods previously described (10). For each marker, forward primers were labeled with fluorescent dyes (6FAM, NED, and Hex) from Applied Biosystems (Foster City, CA) or Integrated DNA Technologies (Coralville, IA). Reverse primers were not labeled. DNA was amplified using MJ Research Tetrad thermocyclers (Waltham, MA) under the following PCR conditions: (i) initial denaturation at $94^{\circ} \mathrm{C}$ for $5 \mathrm{~min}$; (ii) 35 cycles of $94^{\circ} \mathrm{C}$ for $30 \mathrm{~s}, 55$ to $61^{\circ} \mathrm{C}$ (marker dependent) for $30 \mathrm{~s}$, and $72^{\circ} \mathrm{C}$ for $1 \mathrm{~min}$; (iii) $5 \mathrm{~min}$ final extension at $72^{\circ} \mathrm{C}$. PCR products were pooled based on color and size range of the amplified PCR products and the DNA was denatured by heating at $94^{\circ} \mathrm{C}$ for $5 \mathrm{~min}$. PCR products were diluted 200, 500, and 2,000×, and $2 \mu 1$ of the diluted product was added to $9 \mu$ of formamide-containing ROX/LIZ (dependent on the size of the product) labeled size standards (Applied Biosystems). PCR products from different primer pairs having different size ranges and labels were combined for simultaneous analysis using a Mini Prep75 (Tecan Group Ltd., Männedorf, Switzerland) instrument based on the manufacturer protocols, and analyzed to determine the size of the SSR alleles. The reaction was run on an ABI Prism 3730 DNA Analyzer (Applied Biosystems) following manufacturer's instructions. Fragment size and SSR marker genotype analysis were performed with Gene Mapper software version 3.7 (Applied Biosystems). Allele sizes for all SSR markers used in the present study are displayed in Table 1.

\section{RESULTS AND DISCUSSION}

In the present study, a total of 178 rice accessions were initially identified by utilizing the SSR marker RM208 and the dominant marker Pib-dom, which were shown to have strong association with the $P i b$ gene in a previous study (10). A total of 164 of these 178 accessions were verified to match data from the purified core

Races

$\begin{array}{lllllllll}\text { IB1 IB49 } & \text { IB54 IB45 IH1 IG1 IC17 } & \text { IE1 IE1K }\end{array}$

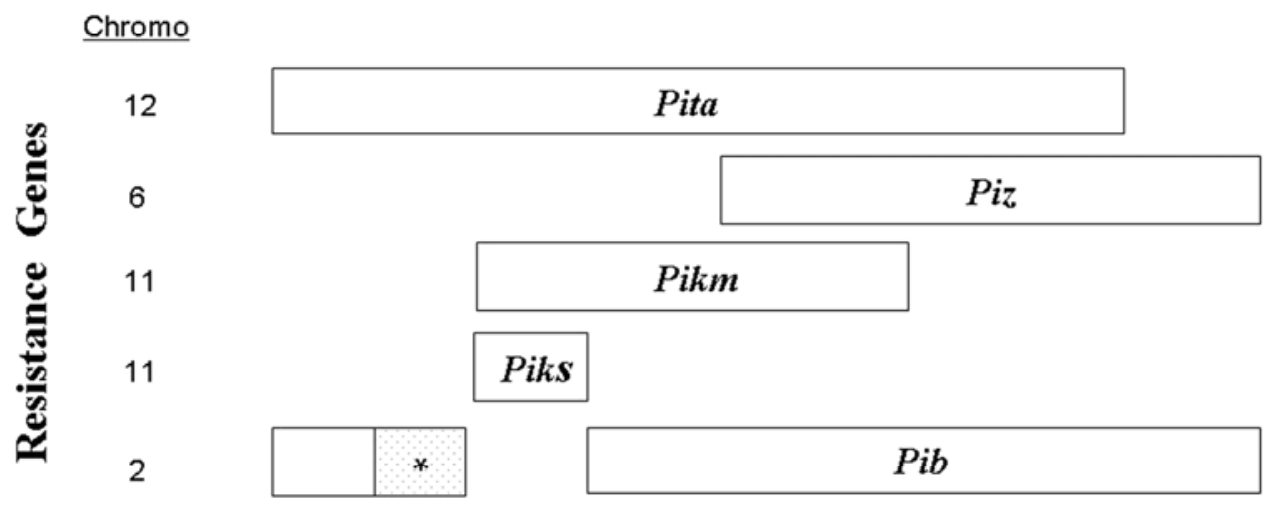

* Partial resistance

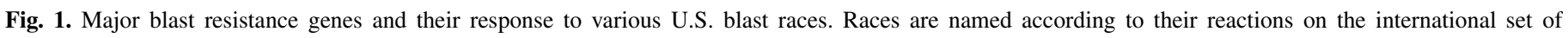
differential cultivars used in Atkins et al (3). 
collection with three high PIC value SSRs and RM208. The remaining 14 accessions were removed due to suspected seed mix. Based on the gene-for-gene theory, Pib should be present only if the germplasm accession is (i) resistant to $A V R$ races, such as IE1k and IB1, and (ii) susceptible to a virulent (VIR) race, such as IB54 (10). Utilizing these differential races, Pib was detected in 128 of 164 germplasm accessions since they were resistant to IE1k and IB1 but susceptible to IB54 (Fig. 1). Out of the 128 accessions with $P i b, 89$ had alleles typically associated with the presence of Pib for all three markers (RM208 [179], RM166 [318], and Pib-dom [360]) examined (Fig. 2; Table 2). The presence of all three $P i b$ marker alleles in these germplasm accessions suggests that they all contain the same Pib haplotype. This was unexpected because these 89 germplasm accessions were collected from several geographic regions of the world, including Central and South America, Europe, Asia, and Africa (Table 2). Although these cultivars are not known to have direct parentage in common, it is still possible that they inherited Pib from the same donor. In contrast, 17 germplasm accessions contained just two of the $P i b$ associated marker alleles, suggesting that these accessions contain different $P i b$ haplotypes. Additionally, 16 germplasm accessions followed the gene-for-gene concept but had no Pib associated marker alleles. Thus, the presence of $P i b$ utilizing our present markers could not be verified in these 16 accessions.

In contrast, 28 germplasm accessions having 0 to 3 Pib marker alleles were found to be resistant to all races, IE-1k, IB54, and IB1, suggesting these accessions contain other $R$ genes that are responsible for resistance to IB54 (such as Pita or Pi-ks) (24) and perhaps IE-1k and IB1. Because of the incongruity between the markers and the race reactions, the presence or absence of Pib cannot be verified in these accessions (Fig. 2) (4). As examples of accessions resistant to all three races, Juma 61 had two Pib associated alleles, T442-57 had one Pib associated allele, and accessions C1-6-5-3 and Hansraj had no Pib associated alleles, indicating that resistant reactions observed were due to other $R$ genes. Moreover, the cultivar Saber carries all three of the Pib resistant alleles and was resistant to IE1k and IB1 as predicted, but was also resistant to IB54 due to the presence of the Pi-km allele (Table 2, Fig. 1) (25).

Finally, a total of four germplasm accessions, R 647 from China, 17465-4 and Bilo from Fiji, and BR-IRGA-410 from Brazil, had all the expected marker alleles for having the Pib gene but were susceptible to all three tested races. One accession RP2199-16-2-2-1 from India having all expected marker alleles for $P i b$ was resistant to IB1, but susceptible to both IE1k and IB54 (Table 2).

Inconsistencies in the marker and phenotype analysis in this study can be attributed to genetic variability among the rice accessions and within the pathogen. Although we used single spore isolates, the rice blast fungus is known to be hyper-variable and thus it is impossible to have near-isogenic isolates (AVR/VIR strains of each race) $(4,5)$ to test the gene for gene concept. MAS can overcome some disadvantages in pathogenicity assays for monitoring $R$ genes; however, the power of MAS is dependent on how reliable the markers are, as the presence of marker alleles sometimes does not indicate the presence of functional $R$ genes $(17,18)$. In this study, five accessions which putatively possessed $P i b$ based on markers were susceptible to all three races, with three of these accessions having all three Pib associated marker alleles. The associated marker alleles in these cases do not indicate the presence of a functional Pib gene, suggesting that marker analysis alone would not work using these five germplasm accessions. These incongruities and others demonstrate that the markers for $P i b$ do not always predict gene function. One reason that the Pib gene may not be expressed is due to a promoter mutation or additional mutations in the $P i b$ gene outside of the region used to design the dominant marker. However, it may take considerable effort to examine promoter and transcript levels for this gene because expression of Pib has been seen to be influenced by diverse environmental factors $(34,35)$. It may be that sequence analysis of the $P i b$ gene coding region in these accessions could infer possible mutations that result in a nonfunctional copy of $P i b$ and allow the development of an improved or functional $P i b$ marker in the future.

TABLE 1. Summary of $P i b$ associated allele sizes, annealing temperatures, and sequences of dominant and simple sequence repeat markers at the $P i b$ locus

\begin{tabular}{lccclc}
\hline Marker & Size (bases) & $\begin{array}{c}\text { Recombination } \\
\text { distance from } \mathrm{Pib}\end{array}$ & $\begin{array}{c}\text { Annealing } \\
\text { temperature }\left({ }^{\circ} \mathrm{C}\right)\end{array}$ & \multicolumn{1}{c}{ Forward primer } & Reverse primer \\
\hline Pib-dom & 360 & 0.0 & 55 & GAACAATGCCCAAACTTGAGA & GGGTCCACATGTCAGTGAGC \\
RM208 & 179 & 0.0 & 55 & TCTGCAAGCCTTGTCTGATG & TAAGTCGATCATTGTGTGGACC \\
RM166 & 316 & 2.3 & 61 & GGTCCTGGGTCAATAATTGGGTACC & TTGCTGCATGATCCTAAACCGG \\
\hline
\end{tabular}

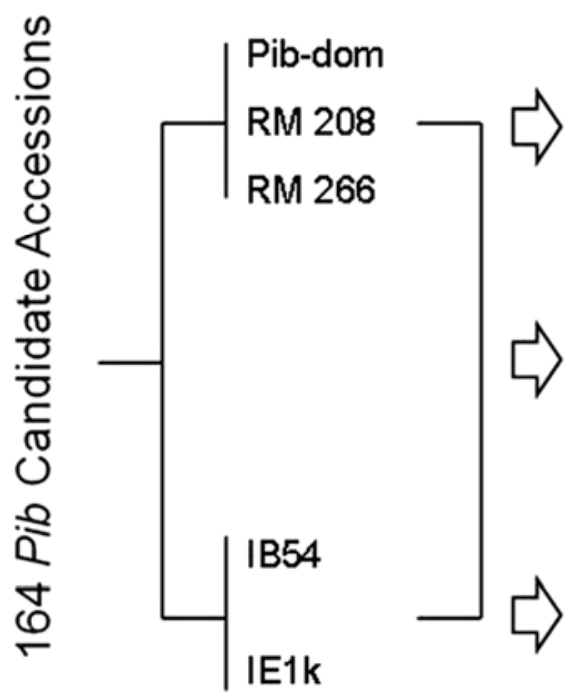

128 IB54-S, IB1 and IE1k-R

$89: 3 \exp$. alleles

17: 2 exp. alleles

3: Pib dom only

3: RM 166 only

16: no exp. alleles

28 IB54-R, IB1 and IE1k-R

$24: 3 \exp$. alleles

$1: 2$ exp. alleles

1: 1 exp. allele

2 : no exp. alleles

8 IB54-S, IB1 and IE1k-S No $R$ genes, or components

$5: 3 \exp$. alleles $\square$ of Pib altered, resulting in

3: no exp. alleles $\sqrt{ }$ non-function

Fig. 2. Analysis of the $P i b$ gene in rice germplasm using disease reaction and simple sequence repeat (SSR) markers. The diagram shows results of disease reactions and expected SSR marker alleles for germplasm in different categories. 


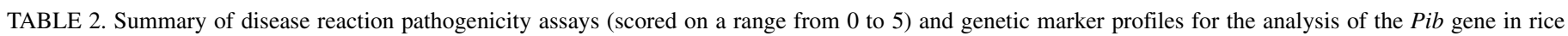
germplasm accessions

\begin{tabular}{|c|c|c|c|c|c|c|c|c|c|}
\hline \multirow[b]{2}{*}{ Sample } & \multirow[b]{2}{*}{ Country } & \multirow[b]{2}{*}{ Name } & \multicolumn{3}{|c|}{ SSR markers $^{\mathrm{a}}$} & \multicolumn{3}{|c|}{ Disease reactions } & \multirow{2}{*}{$\begin{array}{c}\text { Presence } \\
\text { of } P i b^{\mathrm{b}}\end{array}$} \\
\hline & & & Pib-dom & RM208 & RM166 & IB54 & IE1k & IB1 & \\
\hline GSOR310285 & Philippines & IR 532-1-47 & 360 & 179 & 316 & S 3 & R 0 & R 0 & + \\
\hline GSOR310298 & Guyana & 51779 & 360 & 179 & 316 & S 3 & R 2 & R 0 & + \\
\hline GSOR310326 & Philippines & IR $1103-15-8-5-3-3-3$ & 360 & 179 & 316 & S 3 & R 2 & R 0 & + \\
\hline GSOR310340 & Laos & Chao Hay b & 360 & 179 & 316 & S 4 & R 0 & R 0 & + \\
\hline GSOR310363 & Colombia & P773-44-3-1 & 360 & 179 & 316 & S 3 & R 2 & R 0 & + \\
\hline GSOR310367 & Colombia & P 738-97-3-1 & 360 & 179 & 316 & S 3 & R 0 & R 0 & + \\
\hline GSOR310368 & Colombia & P 761-40-2-1 & 360 & 179 & 316 & S 3 & R 0 & R 0 & + \\
\hline GSOR310481 & India & Anandi & 360 & 179 & 316 & S 3 & R 0 & R 0 & + \\
\hline GSOR310485 & Sri Lanka & Perum Karuppan & 360 & 164 & 318 & S 4 & R 2 & R 0 & + \\
\hline GSOR310487 & Indonesia & Sigadis & 360 & 179 & 316 & S 4 & R 0 & R 0 & + \\
\hline GSOR310517 & Hong Kong & Fa Loh Pak & $\times$ & 164 & 316 & S 3 & R 2 & R 0 & $+/ ?$ \\
\hline GSOR310528 & Brazil & J 312 & 360 & 179 & 316 & S 3 & R 0 & R 0 & + \\
\hline GSOR310539 & Mali & Segadis & 360 & 179 & 316 & S 4 & R 0 & R 0 & + \\
\hline GSOR310542 & Bangladesh & BR51-319-9 & 360 & 179 & 316 & S 3 & R 0 & R 0 & + \\
\hline GSOR310545 & Indonesia & B462B-PN-31-2 & 360 & 179 & 316 & S 4 & R 0 & R 0 & + \\
\hline GSOR310547 & Peru & Huallaga & 360 & 179 & 316 & S 3 & R 2 & R 0 & + \\
\hline GSOR310548 & Thailand & BKN 6820-6-3-2 & 360 & 179 & 316 & S 3 & R 2 & R 0 & + \\
\hline GSOR310549 & Sri Lanka & BG 90-2 & 360 & 179 & 316 & S 3 & R 0 & R 0 & + \\
\hline GSOR310567 & Guatemala & Tikal2 & 360 & 179 & 316 & S 3 & R 2 & R 0 & + \\
\hline GSOR310574 & Malaysia & SM II & 360 & 179 & 316 & S 3 & R 2 & R 0 & + \\
\hline GSOR310575 & Haiti & Gros Riz & 360 & 176 & 316 & S 3 & R 2 & R 0 & + \\
\hline GSOR310576 & India & Pusa 33 & 360 & 179 & 316 & S 3 & R 1 & R 0 & + \\
\hline GSOR310612 & Uzbekistan & Uz Begohef 2 & 360 & 179 & 316 & S 3 & R 2 & R 0 & + \\
\hline GSOR310630 & Thailand & BKN 6987-68-14 & 360 & 179 & 316 & S 4 & R 2 & R 0 & + \\
\hline GSOR310631 & Guinea & GPNO 22236 & 360 & 179 & 316 & S 4 & R 2 & R 0 & + \\
\hline GSOR310632 & Philippines & IR 4482-5-3-9-5 & 360 & 179 & 316 & S 3 & R 2 & R 0 & + \\
\hline GSOR310636 & Cote D'Ivoire & IRAT 8 & 360 & 179 & 316 & S 3 & R 0 & R 0 & + \\
\hline GSOR310650 & India & PR 106 & 360 & 179 & 316 & S 3 & R 0 & R 0 & + \\
\hline GSOR310655 & Chile & CH 272-132 & 360 & 179 & 316 & S 3 & R 0 & R 0 & + \\
\hline GSOR310657 & Egypt & CR418-3-12 & 360 & 179 & 316 & S 3 & R 0 & R 0 & + \\
\hline GSOR310658 & Egypt & CR 561-4-2-1 & 360 & 179 & 318 & S 4 & R 2 & R 0 & + \\
\hline GSOR310663 & Kazakhstan & Kasakstanica & 360 & 164 & 318 & S 3 & R 0 & R 0 & + \\
\hline GSOR310690 & S. Korea & Milyang23/IR1545-339-2-2 & 360 & 179 & 316 & S 3 & R 0 & R 0 & + \\
\hline GSOR310709 & Bangladesh & BR19 & 360 & 179 & 316 & S 3 & R 0 & R 0 & + \\
\hline GSOR310732 & Colombia & C 3CU77-1CU-2CU-2CU-2CU-SMCU2 & 360 & 179 & 316 & S 3 & R 0 & R 0 & + \\
\hline GSOR310735 & Panama & Anayansi & 360 & 179 & 316 & S 3 & R 0 & R 0 & + \\
\hline GSOR310741 & Cuba & Perla & 360 & 179 & 316 & S 3 & R 0 & R 0 & + \\
\hline GSOR310746 & Cambodia & 376 & 360 & 179 & 316 & S 4 & R 2 & R 0 & + \\
\hline GSOR310748 & Nepal & IR-44595 & 360 & 176 & 316 & S 3 & R 0 & R 0 & + \\
\hline GSOR310757 & India & RP2151-173-1-8 & 360 & 179 & 316 & S 3 & R 2 & R 0 & + \\
\hline GSOR310770 & China & Miyang & 360 & 179 & 316 & S 4 & R 0 & R 0 & + \\
\hline GSOR310773 & Cuba & ECIA76-S89-1 & 360 & 179 & 316 & S 3 & R 0 & R 0 & + \\
\hline GSOR311005 & Philippines & IR 8-296-2-1 & 360 & 179 & 316 & S 3 & R 0 & R 0 & + \\
\hline GSOR311024 & India & RP1 332 & 360 & 179 & 316 & S 3 & R 2 & R 0 & + \\
\hline GSOR311032 & Guyana & 50638 & 360 & 179 & 316 & S 3 & R 0 & R 0 & + \\
\hline GSOR311033 & Argentina & Fortuna Corrientes Sel Inta & 360 & 179 & 421 & S 3 & R 0 & R 0 & + \\
\hline GSOR311042 & Philippines & IR 1314-28-1-2 & 360 & 179 & 316 & S 3 & R 0 & R 0 & + \\
\hline GSOR311061 & Philippines & Siryan & 360 & 179 & 316 & S 4 & R 0 & R 0 & + \\
\hline GSOR311066 & Laos & Kh. Mack Fay & 360 & 179 & 316 & S 3 & R 0 & $\mathrm{R} 0$ & + \\
\hline GSOR311073 & Indonesia & Tukan Tuna & 360 & 179 & $x$ & S 3 & R 0 & R 0 & + \\
\hline GSOR311076 & Bulgaria & Sesilla & 360 & 179 & 316 & S 3 & R 0 & R 0 & + \\
\hline GSOR311113 & Hong Kong, China & Shui Ya Jien & 360 & 179 & 316 & S 3 & R 0 & R 0 & + \\
\hline GSOR311152 & Fiji & Rani & 360 & 179 & 316 & S 3 & R 0 & R 0 & + \\
\hline GSOR311153 & Philippines & IR 2061-214-2-3 & 360 & 176 & 316 & S 3 & R 0 & R 0 & + \\
\hline GSOR311154 & Philippines & IR2151-598-3-5 & 360 & 179 & 316 & S 3 & R 0 & R 0 & + \\
\hline GSOR311162 & Guyana & $60-283$ & 360 & 179 & 318 & S 3 & R 2 & R 0 & + \\
\hline GSOR311168 & Philippines & IR9-60 & 360 & 179 & 316 & S 3 & R 0 & R 0 & + \\
\hline GSOR311184 & Thailand & Bang Tuey & 360 & 176 & 316 & S 4 & R 0 & R 0 & + \\
\hline GSOR311207 & India & NP 97 & $x$ & 164 & 316 & S 3 & R 2 & $\mathrm{R} 0$ & $+/ ?$ \\
\hline GSOR311210 & Philippines & IR 2151-745-3-1 & 360 & 179 & 316 & S 3 & R 2 & R 0 & + \\
\hline GSOR311213 & Bangladesh & Biplab & 360 & 179 & 316 & S 3 & R 2 & R 0 & + \\
\hline GSOR311214 & Philippines & IR 1514A-E597 & 360 & 176 & 318 & S 3 & R 1 & R 0 & + \\
\hline GSOR311217 & Pakistan & Sella Manzkhora & 360 & 179 & 316 & S 3 & R 0 & R 0 & + \\
\hline GSOR311223 & Indonesia & KN-1 B-361-BLK-2 & 360 & 172 & 316 & S 3 & R 0 & R 0 & + \\
\hline GSOR311238 & Sierra Leone & Chen Chu Ai & 360 & 179 & $x$ & S 3 & R 1 & R 0 & + \\
\hline GSOR311248 & Dominican Republic & Mingolo & 360 & 179 & 316 & S 4 & R 0 & R 0 & + \\
\hline \multirow[t]{2}{*}{ GSOR311249 } & Dominican Republic & Tono Brea 439 & 360 & 179 & 316 & S 5 & R 0 & R 0 & + \\
\hline & & & & & & & \multicolumn{3}{|c|}{ (continued on following page) } \\
\hline
\end{tabular}

${ }^{\mathrm{a}} \times$ indicates lack of amplification product detected for simple sequence repeat (SSR) marker.

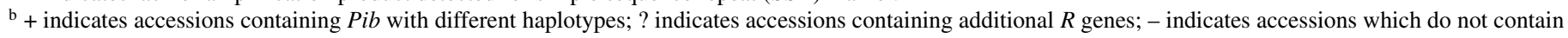
$P i b$; and * indicates accessions with no marker alleles with pathogenicity response similar to accessions containing $P i b$. 


\begin{tabular}{|c|c|c|c|c|c|c|c|c|c|}
\hline \multirow[b]{2}{*}{ Sample } & \multirow[b]{2}{*}{ Country } & \multirow[b]{2}{*}{ Name } & \multicolumn{3}{|c|}{ SSR markers ${ }^{\mathrm{a}}$} & \multicolumn{3}{|c|}{ Disease reactions } & \multirow{2}{*}{$\begin{array}{c}\text { Presence } \\
\text { of } P i b^{\mathrm{b}}\end{array}$} \\
\hline & & & Pib-dom & RM208 & RM166 & IB54 & IE1k & IB1 & \\
\hline GSOR311294 & Senegal & CAS 209 & 360 & 179 & 316 & S 3 & R 2 & R 0 & + \\
\hline GSOR311298 & Thailand & Jek Chuey 159 & 360 & 179 & 316 & S 3 & R 2 & R 0 & + \\
\hline GSOR311302 & Sierra Leone & SL 22-613 & 360 & 179 & 421 & S 4 & R 2 & R 0 & + \\
\hline GSOR311306 & Nigeria & Mange2 & 360 & 179 & 316 & S 3 & R 0 & R 0 & + \\
\hline GSOR311310 & India & Archana & 360 & 179 & 316 & S 4 & R 0 & $\mathrm{R} 0$ & + \\
\hline GSOR311317 & Philippines & IR $1615-246$ & 360 & 179 & 318 & S 3 & R 0 & $\mathrm{R} 0$ & + \\
\hline GSOR311325 & Italy & Bajang Allorio & 360 & 179 & 316 & S 3 & R 0 & R 0 & + \\
\hline GSOR311344 & Philippines & IR 9209-26-2 & 360 & 179 & 316 & $\mathrm{~S} 4$ & $\mathrm{R} 2$ & R 0 & + \\
\hline GSOR311348 & S. Korea & Seogwangbyeo & 360 & 179 & 316 & S 3 & R 0 & R 0 & + \\
\hline GSOR311359 & Colombia & 17632 & 360 & 179 & 316 & S 3 & R 0 & R 0 & + \\
\hline GSOR311360 & Colombia & 19965 & 360 & 179 & 316 & S 3 & R 0 & R 0 & + \\
\hline GSOR311366 & China & Te Qing & 360 & 179 & 316 & S 3 & R 0 & R 0 & + \\
\hline GSOR311380 & Bangladesh & BR24 & 360 & 179 & 316 & S 3 & R 0 & R 0 & + \\
\hline GSOR311399 & Colombia & Amistad 82 & 360 & 179 & 316 & S 3 & R 0 & R 0 & + \\
\hline GSOR311402 & Ecuador & INIAP 11 & 360 & 179 & 316 & S 4 & R 0 & R 0 & + \\
\hline GSOR311403 & Colombia & Panama 1048 & 360 & 179 & 316 & S 3 & R 0 & R 0 & + \\
\hline GSOR311405 & Colombia & Huri 282 & 360 & 179 & 316 & S 3 & R 0 & R 0 & + \\
\hline GSOR311423 & Philippines & IR 58614-B-B-8-2 & 360 & 176 & 316 & S 3 & R 0 & R 0 & + \\
\hline GSOR311424 & Japan & BL 1 & 360 & 179 & 418 & S 3 & R 0 & R 0 & + \\
\hline GSOR311430 & Cuba & ECIA 66 & 360 & 179 & 316 & S 3 & R 2 & $\mathrm{R} 0$ & + \\
\hline GSOR311433 & Philippines & IR 54055-142-2-1-2-3 & 360 & 179 & 316 & S 4 & R 0 & R 0 & + \\
\hline GSOR311435 & Vietnam & CM1, Haipong & 360 & 179 & 316 & S 3 & R 0 & R 0 & + \\
\hline GSOR311438 & Liberia & $2071-621-2$ & 360 & 179 & 316 & S 3 & R 2 & $\mathrm{R} 0$ & + \\
\hline GSOR311439 & China & 4582 & 360 & 172 & 316 & S 3 & R 0 & $\mathrm{R} 0$ & + \\
\hline GSOR311441 & China & GP-2 & 360 & 179 & 316 & S 3 & R 0 & R 0 & + \\
\hline GSOR311442 & Philippines & IR58025 B & 360 & 179 & 316 & S 3 & R 0 & $\mathrm{R} 0$ & + \\
\hline GSOR311443 & China & Gui 99 & 360 & 179 & 316 & S 3 & R 0 & $\mathrm{R} 0$ & + \\
\hline GSOR311445 & China & Z 535 & 360 & 179 & 316 & S 4 & R 0 & $\mathrm{R} 0$ & + \\
\hline GSOR311447 & China & Xiangzhaoxian NO. 15 & 360 & 179 & 316 & S 4 & R 0 & R 0 & + \\
\hline GSOR311448 & China & Hunanruanmi & 360 & 179 & 316 & S 3 & R 0 & R 0 & + \\
\hline GSOR311449 & China & Zhongyu No. 6 & 360 & 179 & 316 & S 3 & R 0 & $\mathrm{R} 0$ & + \\
\hline GSOR311456 & China & Erxi No. 149 & 360 & 179 & 316 & S 3 & R 0 & R 0 & + \\
\hline GSOR311459 & China & 71198 & 360 & 179 & 316 & S 3 & R 0 & R 0 & + \\
\hline GSOR311467 & China & Jinnuo No. 6 & 360 & 176 & 316 & S 3 & R 2 & R 0 & + \\
\hline GSOR311468 & China & Dian No. 01 & $\times$ & 172 & 316 & S 3 & R 0 & R 0 & $+/ ?$ \\
\hline GSOR311471 & China & You No. 51 & 360 & 179 & 316 & S 3 & R 0 & R 0 & + \\
\hline GSOR311477 & China & Н 323 & 360 & 179 & 316 & S 3 & R 0 & $\mathrm{R} 0$ & + \\
\hline GSOR311478 & China & CDR 22 & 360 & 179 & $\times$ & S 3 & R 0 & R 0 & + \\
\hline GSOR311481 & China & Shufeng 121 & 360 & 179 & 316 & S 3 & R 0 & R 0 & + \\
\hline GSOR311511 & China & MPH 501 & 360 & 179 & 316 & S 3 & R 0 & R 0 & + \\
\hline GSOR311518 & Bangladesh & Bhujon Kolpo & 360 & 179 & 316 & S 3 & R 0 & R 0 & + \\
\hline GSOR311519 & Bangladesh & Khoia & 360 & 179 & 316 & S 3 & R 0 & R 0 & + \\
\hline GSOR311520 & Bangladesh & Bogra & 360 & 179 & 316 & S 3 & R 0 & R 0 & + \\
\hline GSOR311521 & Philippines & IR 56450-28-2-2 & 360 & 179 & 316 & S 3 & R 0 & R 0 & + \\
\hline \multirow[t]{2}{*}{ GSOR311525 } & Indonesia & S972B-22-1-3-1-1 & 360 & 179 & 316 & S 4 & R 0 & R 0 & + \\
\hline & United States & Saber & 360 & 179 & 316 & R 0 & R0 & $\mathrm{R} 0$ & + \\
\hline GSOR310164 & Mexico & C1-6-5-3 & $\times$ & 164 & $\times$ & R 0 & R 0 & $\mathrm{R} 0$ & $?$ \\
\hline GSOR310350 & Papua New Guinea & C 8435 & 360 & 179 & 316 & R 1 & R 0 & $\mathrm{R} 0$ & $?$ \\
\hline GSOR310540 & Thailand & $\mathrm{T} 442-57$ & $\times$ & 176 & 316 & R 1 & R 0 & $\mathrm{R} 0$ & $?$ \\
\hline GSOR310543 & Costa Rica & CR 1113 & 360 & 179 & 316 & R 1 & R 2 & $\mathrm{R} 0$ & $?$ \\
\hline GSOR310566 & Ecuador & INIAP 7 & 360 & 179 & 316 & R 1 & R 2 & R 0 & $?$ \\
\hline GSOR310648 & Zimbabwe & IR 400 & 360 & 179 & 316 & R 0 & R 0 & $\mathrm{R} 0$ & $?$ \\
\hline GSOR310687 & Philippines & IR 9660-48-1-1-2 & 360 & 179 & 316 & R 1 & R 2 & R 0 & $?$ \\
\hline GSOR310688 & S. Korea & Milyang 56 & 360 & 179 & 316 & R 1 & R 0 & R 0 & $?$ \\
\hline GSOR310689 & S. Korea & Raegyeong & 360 & 179 & 316 & R 1 & R 0 & $\mathrm{R} 0$ & $?$ \\
\hline GSOR310730 & Dominican Republic & Juma 61 & 360 & $\times$ & 316 & R 0 & R 0 & R 0 & $?$ \\
\hline GSOR310750 & Nigeria & Faro 37 & 360 & 179 & 316 & R 0 & R 0 & R 0 & $?$ \\
\hline GSOR310751 & India & RP1821-5-17-2 & 360 & 179 & 316 & R 0 & R 0 & R 0 & $?$ \\
\hline GSOR310752 & Cuba & ECIA 128 & 360 & 179 & 316 & R 0 & R 0 & $\mathrm{R} 0$ & $?$ \\
\hline GSOR310753 & Egypt & GZ1368-5-4 & 360 & 179 & 316 & R 0 & R 0 & $\mathrm{R} 0$ & $?$ \\
\hline GSOR310756 & Dominican Republic & J355-6-2-1-1 & 360 & 179 & 316 & R 0 & R 0 & R 0 & $?$ \\
\hline GSOR310772 & Brazil & CL Seleccion 56 & 360 & 179 & 316 & R 0 & R 1 & R 0 & $?$ \\
\hline GSOR311039 & Philippines & IR 1321-19 & 360 & 179 & 316 & R 0 & R 0 & $\mathrm{R} 0$ & $?$ \\
\hline GSOR311044 & Philippines & IR 773A1-36-2-1-3 & 360 & 179 & 316 & R 0 & R 0 & R 0 & $?$ \\
\hline GSOR311082 & Pakistan & Hansraj & $\times$ & 164 & $\times$ & R 0 & R 0 & R 0 & $?$ \\
\hline GSOR311097 & Portugal & Indo Yiaia Lonica & 360 & 179 & 316 & R 0 & R 0 & $\mathrm{R} 0$ & $?$ \\
\hline GSOR311219 & S. Korea & Suweon 258 & 360 & 179 & 316 & R 1 & R 0 & R 0 & $?$ \\
\hline GSOR311244 & Peru & INTI & 360 & 179 & 316 & R 0 & R 0 & R 0 & $?$ \\
\hline GSOR311304 & Nigeria & Adny 11 & 360 & 179 & 316 & R 0 & R 0 & R 0 & $?$ \\
\hline GSOR311409 & Mexico & Campeche A 80 & 360 & 179 & 316 & R 1 & R 0 & $\mathrm{R} 0$ & $?$ \\
\hline GSOR311411 & Peru & San Martin 86 & 360 & 179 & 316 & R 1 & R 0 & R 0 & $?$ \\
\hline GSOR311421 & Philippines & C2764-10-2 & 360 & 179 & 316 & R 1 & R 0 & R 0 & $?$ \\
\hline GSOR311503 & China & Zhong 413 & 360 & 179 & 316 & R 0 & R 0 & $\mathrm{R} 0$ & $?$ \\
\hline
\end{tabular}




\begin{tabular}{|c|c|c|c|c|c|c|c|c|c|}
\hline \multirow[b]{2}{*}{ Sample } & \multirow[b]{2}{*}{ Country } & \multirow[b]{2}{*}{ Name } & \multicolumn{3}{|c|}{ SSR markers ${ }^{\mathrm{a}}$} & \multicolumn{3}{|c|}{ Disease reactions } & \multirow{2}{*}{$\begin{array}{c}\text { Presence } \\
\text { of } P i b^{\mathrm{b}}\end{array}$} \\
\hline & & & Pib-dom & RM208 & RM166 & IB54 & IE1k & IB1 & \\
\hline GSOR311513 & China & ZAO 402 & 360 & 179 & 316 & R 0 & R 0 & R 0 & $?$ \\
\hline GSOR310278 & Iraq & Amber 33 & $\times$ & 168 & 318 & S 3 & R 0 & $\mathrm{R} 2$ & $*$ \\
\hline GSOR310319 & India & BC5-55 & $x$ & 164 & 318 & S 3 & R 2 & R 0 & $*$ \\
\hline GSOR310352 & Malaysia & Padi Bangka & $x$ & 164 & 418 & S 3 & R 0 & R 0 & $*$ \\
\hline GSOR310436 & Cuba & Zayas Bazan & $x$ & 176 & 318 & S 3 & R 0 & R 0 & $*$ \\
\hline GSOR310553 & Iran & 205 & $x$ & 170 & 318 & S 3 & R 2 & $\mathrm{R} 0$ & $*$ \\
\hline GSOR310555 & Colombia & Colombia 1 & $x$ & 176 & 318 & S 5 & R 1 & R 0 & $*$ \\
\hline GSOR310659 & Egypt & YNA 223 & $\times$ & 168 & 418 & S 3 & R 0 & R 0 & $*$ \\
\hline GSOR310683 & Nigeria & IITA 130 & $x$ & 164 & 421 & S 3 & R 0 & $\mathrm{R} 0$ & $*$ \\
\hline GSOR310686 & Brazil & Pratao & $x$ & 164 & 418 & S 3 & R 0 & R 0 & $*$ \\
\hline GSOR310754 & Argentina & H232-44-1-1 & $x$ & 164 & 418 & S 3 & R 2 & $\mathrm{R} 0$ & $*$ \\
\hline GSOR310856 & China & WC 521 & $x$ & 164 & 418 & S 3 & R 1 & R 0 & $*$ \\
\hline GSOR311059 & Philippines & IR 1103-49-4-1-3-3-2 & $x$ & 164 & 418 & S 3 & R 0 & R 0 & $*$ \\
\hline GSOR311239 & Brazil & Pratao Tipo Guedes & $x$ & 164 & 421 & S 4 & R 0 & R 0 & $*$ \\
\hline GSOR311262 & Zaire & $\mathrm{R} 46 / 3$ & $x$ & 164 & 421 & S 3 & R 0 & R 0 & $*$ \\
\hline GSOR311264 & Zaire & Sechele & $x$ & 164 & 421 & S 3 & R 2 & R 0 & $*$ \\
\hline GSOR311436 & China & Zhongyu No.1 & $x$ & 172 & 318 & S 3 & R 0 & R 0 & $*$ \\
\hline GSOR310583 & Fiji & $17465-4$ & 360 & 179 & 316 & S 3 & S 4 & S 3 & - \\
\hline GSOR310668 & Azerbaijan & Bak Saly Mestnyj & $x$ & 164 & 418 & S 3 & S 3 & $\mathrm{~S} 4$ & - \\
\hline GSOR310685 & Brazil & BR-IRGA-410 & 360 & 179 & 316 & S 3 & S 3 & S 3 & - \\
\hline GSOR311253 & Fiji & Bilo & 360 & 179 & 316 & S 3 & S 3 & S 3 & - \\
\hline GSOR311494 & China & $\mathrm{R} 647$ & 360 & 179 & 316 & S 5 & S 3 & S 3 & - \\
\hline GSOR311524 & India & RP2199-16-2-2-1 & 360 & 179 & 316 & S 3 & S 3 & $\mathrm{R} 0$ & - \\
\hline GSOR311640 & India & ARC 10378 & $x$ & 176 & 418 & S 5 & S 3 & S 4 & - \\
\hline GSOR311668 & Pakistan & Daudzai Field Mix & $x$ & 164 & 318 & S 5 & S 3 & $\mathrm{~S} 4$ & - \\
\hline
\end{tabular}

The use of multiple markers linked with various $R$ genes can be used to determine the basis of resistance in diverse germplasm collections. The Pib-dom marker was derived from a portion of the Pib gene; however, it was not a perfect functional marker (10). In the present study, the Pib-dom marker appeared to be the most accurate, having the least number of disagreements $(=7)$ between its presence and the race reactions expected with the presence of the Pib gene. RM208 and RM166 had 18 and 13 disagreements, respectively. Three of the disagreements between both the Pibdom and RM208 markers and the disease reactions may be the result of other $R$ genes being present besides Pib (indicated in Table 2 with " $+/$ ?" designations) that confer resistance to races IE1k and IB1. In conclusion, no single SSR marker allele was completely $(100 \%)$ associated with the Pib-dom marker or with the presence of $P i b$ as indicated by race reactions.

In the present study, rice germplasm with $P i b$ was found in 40 countries, but predominantly from China, the Philippines, Colombia, and India (Table 2). In the United States, Pib is useful because it confers resistance to IE1k, a virulent race that has overcome resistance mediated by Pita. Field isolates that belong to IE1k have been found in commercial rice fields in the southern United States for decades (18). Stacking Pib or Piz with Pita in advanced breeding lines would achieve more durable resistance to blast (5).

In summary, we not only verified the $P i b$ gene in 109 rice germplasm accessions but also demonstrated the usefulness of combining DNA markers and pathogenicity assays to confirm the presence of $R$ genes (10). The pathogenicity assays helped verify the accuracy of DNA markers and also identified germplasm accessions that may possibly have additional blast $R$ genes.

\section{ACKNOWLEDGMENTS}

We thank M. Lin, L. Bernhardt, A. Jackson, and E. McWhirter as well as other staff members of DB NRRC for their technical assistance. For germplasm requests, please visit www.ars.usda.gov/spa/dbnrrc/gsor at GSOR of DB NRRC. The U.S. Department of Agriculture is an equal opportunity provider and employer.

\section{LITERATURE CITED}

1. Agrama, H. A., Yan, W., Lee, F., Fjellstrom, R., Chen, M. H., and McClung, A. 2009. Genetic assessment of a mini-core subset developed from the USDA rice genebank. Crop Sci. 49:1336-1346.

2. Ashikawa, I., Hayashi, N., Yamane, H., Kanamori, H., Wu, J., Matsumoto, T., Ono, K., and Yano, M. 2008. Two adjacent nucleotide-binding siteleucine-rich repeat class genes are required to confer Pikm-specific rice blast resistance. Genetics 180:2267-2276.

3. Atkins, J. G., Robert, A. L., Adair, C. R., Goto, K., Kozaka, T., Yanagida, R., Yamada, M., and Matsumoto, S. 1967. An international set of rice varieties for differentiating races of Pyricularia oryzae. Phytopathology 57:297-301.

4. Babujee, L., and Gnanamanickam, S. S. 2000 Molecular tools for characterization of rice blast pathogen (Magnaporthe grisea) population and molecular marker-assisted breeding for disease resistance. Curr. Sci. 78:248-257

5. Bonman, J. M., Khush, G. S., and Nelson, R. J. 1992. Breeding rice for resistance to pests. Annu. Rev. Phytopathol. 30:507-528.

6. Bryan, G. T., Wu, K. S., Farrall, L., Jia, Y., Hershey, H. P., McAdams, S. A., Faulk, K. N., Donaldson, G. K., Tarchini, R., and Valent, B. 2000. A single amino acid difference distinguishes resistant and susceptible alleles of the rice blast resistance gene Pi-ta. Plant Cell 12:2033-2045.

7. Chen, X., Shang, J., Chen, D., Lei, C., Zou, Y., Zhai, W., Liu, G., Xu, J., Ling, Z., Cao, G., Ma, B., Wang, Y., Zhao, X., Li, S., and Zhu, L. A. 2006. B-lectin receptor kinase gene conferring rice blast resistance. Plant J. 46:794-804.

8. Costanzo, S., and Jia, Y. 2010. Sequence variation at the rice blast resistance gene Pi-km locus: Implications for the development of allele specific markers. Plant Sci. 178:523-530.

9. Ebron, L. A., Fukuta, Y., Imbe, T., Kato, H., Yanoria, J. M. T., Tsunematsu, H., Khush, G. S., and Yokoo, M. 2004. Estimation of genes in blast resistance in elite indica-type rice (Oryza sativa $\mathrm{L}$.) varieties bred at the International Rice Research Institute. Breed. Sci. 54:381-387.

10. Fjellstrom, R. G., Conaway-Bormans, C. A., McClung, A. M., Marchetti, M. A., Shank, A. R., and Park, W. D. 2004. Development of DNA markers suitable for marker assisted selection of three Pi genes conferring resistance to multiple Pyricularia grisea pathotypes. Crop Sci. 44:1790-1798.

11. Flor, H. H. 1971. Current status of the gene-for-gene concept. Annu. Rev. Phytopathol. 9:275-296.

12. Fukuoka, S., Saka, N., Koga, H., Ono, K., Shimizu, T., Ebana, K., Hayashi, N., Takahashi, A., Hirochika, H., Okuno, K., and Yano, M. 2009. Loss of function of a proline-containing protein confers durable disease resistance in rice. Science 325:998-1001.

13. Harushima, Y., Yano, M., Shomura, A., Sato, M., Shimano, T., Kuboki, Y., Yamamoto, T., Lin, S. Y., Anonio, B. A., Parco, A., Kajiya, H., Huang, N., 
Yamamoto, K., Nagamura, Y., Kurata, N., Khush, G. S., and Sasaki, T. 1998. A high-density rice genetic linkage map with 2275 markers using a single F2 population. Genetics 148:479-494.

14. Hayashi, K., Hashimoto, N., Daigen, M., and Ashikawa, I. 2004. Development of PCR-based SNP markers for rice blast resistance genes at the Piz locus. Theor. Appl. Genet. 108:1212-1220.

15. Hayashi, K., Yasuda, N., Fujita, Y., Koizumi, S., and Yoshida, H. 2010. Identification of the blast resistance gene Pit in rice cultivars using functional markers. Theor. Appl. Genet. 121:1357-1367

16. Hayashi, N., Inoue, H., Kato, T., Funao, T., Shirota, M., Shimizu, T., Kanamori, H., Yamane, H., Hayano-Saito, Y., Matsumoto, T., Yano, M., and Takatsuji, H. 2010. Durable panicle blast-resistance gene $\mathrm{Pbl}$ encodes an atypical CC-NBS-LRR protein and was generated by acquiring a promoter through local genome duplication. Plant J. 64:498-510.

17. Hittalmani, S., Parco, A., Mew, T. V., Zeigler, R. S., and Huang, N. 2000. Fine mapping and DNA marker-assisted pyramiding of the three major genes for blast resistance in rice. Theor. Appl. Genet. 100:1121-1128.

18. Jia, Y., Liu, G., Costanzo, S., Lee, S., and Dai, Y. 2009. Current progress on genetic interactions of rice with rice blast and sheath blight fungi. Frontiers Agric. China 3:231-239.

19. Kiyosawa, S. 1972. The inheritance of blast resistance transferred from some indica varieties of rice. Bull. Natl. Inst. Agric. Sci. D23:69-95.

20. Kiyosawa, S. 1982. Genetics and epidemiological modeling of breakdown of plant disease resistance. Annu. Rev. Phytopathol. 20:93-117.

21. Lee, S. K., Song, M. Y., Seo, Y. S., Kim, H. K., Ko, S., Cao, Suh, J. P., Yi, G., Roh, J. H., Lee, S., An, G., Hahn, T. R., Wang, G. W., Ronald, P., and Jeon, J. S. 2009. Rice Pi5-mediated resistance to Magnaporthe oryzae requires the presence of two coiled-coil-nucleotide-binding-leucine-rich repeat genes. Genetics 181:1627-1638.

22. Lin, F., Chen, S., Que, Z., Wang, L., Liu, X., and Pan, Q. 2007. The blast resistance gene Pi37 encodes a nucleotide binding site-leucine-rich repeat protein and is a member of a resistance gene cluster on rice chromosome 1. Genetics 177:1871-1880.

23. Liu, X., Lin, F., Wang, L., and Pan, Q. 2007. The in-silico map-based cloning of Pi36, a rice coiled-coil-nucleotide-binding site-leucine-rich repeat gene that confers race-specific resistance to the blast fungus. Genetics 176:2541-2549.

24. Marchetti, M. A., Lai, X., and Bollich, C. N. 1987. Inheritance of resistance to Pyricularia oryzae in rice cultivars grown in the United States. Phytopathology 77:799-804.

25. McClung, A. M., Fjellstrom, R. G., Bergman, C. J., Bormans, C. A., Park, W. D., and Marchetti, M. A. 2004. Registration of 'Saber' rice. Crop Sci. 44:693-694.

26. Miyamoto, M., Ando, I., Rybka, K., Kodama, O., and Kawasaki, S. 1996. High resolution mapping of the indica-derived rice blast resistance genes. I. Pib. Mol. Plant-Microbe Interact. 9:6-13.
27. Monna, L., Miyao, A., Zhong, H. S., Yano, M., Iwamoto, M., Umehara, Y., Kurata, N., Hayasaka, H., and Sasaki, T. 1997. Saturation mapping with subclones of YACs: DNA marker production targeting the rice blast disease resistance gene, Pib. Theor. Appl. Genet. 94:170-176.

28. Noda, T., Hayashi, N., Du, P. V., Dinh, H. D., and Lai Van, E. 1999. Distribution of pathogenic races of rice blast fungus in Vietnam. Ann. Phytopathol. Soc. Jpn. 65:526-530.

29. Qu, S., Liu, G., Zhou, B., Bellizzi, M., Zeng, L., Dai, L., Han, B., and Wang, G. W. 2006. The broad-spectrum blast resistance gene Pi9 encodes a nucleotide-binding site-leucine-rich repeat protein and is a member of a multigene family in rice. Genetics 172:1901-1914.

30. RoyChowdhuy, M., Jia, Y., Jackson, A., Jia, M. H., Fjellstrom, R., and Cartwright, R. 2011. Analysis of rice blast resistance gene Pi-z using pathogenicity assays and DNA markers. Euphytica 184:35-46.

31. Shang, J., Tao, Y., Chen, X., Zou, Y., Lei, C., Wang, J., Li, X., Zhao, X., Zhang, M., Lu, Z., Xu, J., Chen, Z., Wan, J., and Zhu, L. 2009. Identification of a new rice blast resistance gene, Pid3, by genome-wide comparison of paired nucleotide-binding site-leucine-rich repeat genes and their pseudogene alleles between the two sequenced rice genomes. Genetics 182:1303-1311.

32. Silue, D., and Notteghem, J. L. 1990. Production of perithecia of Magnaporthe grisea on rice plants. Mycol. Res. 94:1151-1152.

33. Valent, B., Farrall, L., and Chumley, F. G. 1991. Magnaporthe grisea genes for pathogenicity and virulence identified through a series of backcrosses. Genetics 127:87-101.

34. Wang, Z., Yamanouchi, U., Katayose, Y., Sasaki, T., and Yano, M. 2001. Expression of the $P i b$ rice-blast-resistance gene family is up-regulated by environmental conditions favouring infection and by chemical signals that trigger secondary plant defences. Plant Mol. Biol. 47:653-661.

35. Wang, Z. X., Yano, M., Yamanouchi, U., Iwamoto, M., Monna, L., Hayasaka, H., Katayose, Y., and Sasaki, T. 1999. The Pib gene for rice blast resistance belongs to the nucleotide binding and leucine-rich repeat class of plant disease resistance genes. Plant J. 19:55-64.

36. Xin, Z., Velten, J. P., Oliver, M. J., and Burke, J. J. 2003. High-throughput DNA extraction method suitable for PCR. Biotechnology 34:820-825.

37. Yan, W., Rutger, J. N., Bockelman, H. E., Fjellstrom, R. G., Chen, M. H., Tai, T., and McClung, A. M. 2007. Development and evaluation of a core subset of the USDA rice (Oryza sativa L.) germplasm collection. Crop Sci. 47:869-878.

38. Yokoo, M. 2005. Introduction of Piz-t and Pib genes for blast disease resistance from indica varieties and their utilization for genetic researches in rice. Japanese Agric. Res. Q. 39:239-245.

39. Zhou, B., Qu, S., Liu, G., Dolan, M., Sakai, H., Lu, G., Bellizzi, M., and Wang, G. L. 2006. The eight amino-acid differences within three leucinerich repeats between $\mathrm{Pi} 2$ and $\mathrm{Piz}-\mathrm{t}$ resistance proteins determine the resistance specificity to Magnaporthe grisea. Mol. Plant-Microbe Interact. 19:1216-1228 